\title{
THE GROWING MENACE OF ANTIBIOTICS MISUSE IN PAKISTAN
}

\section{Dr. Sofia Shehzad}

'Use and abuse' of antibiotics has long been recognized as a challenge confronting many developed and developing nations. While the more vigilant nations have been able to address the issue through strict drug control regulations, countries like Pakistan continue to struggle with the menace of improper use of medicines specially the anti infective agents. Impact of this hazardous trend on the health and economy of our society tends to go un-noticed and the public, health officials as well as media officials seem to be either least interested or completely unaware of the implications involved.

Antibiotics are essential drugs which appear to change, for better or worse, the nature of several disorders, including bowel conditions, metabolic disorders or liver disease ${ }^{1}$. They are known to reduce morbidity from a variety of bacterial infections and are potential life savers in certain florid septic conditions such as bacteremia and meningitis. Their inappropriate use, however has led to decreased efficacy and bacterial resistance, thereby undermining their useful potential. The later effect i.e. resistance of certain bacterium to respond to drugs they are usually susceptible to, results from a gene mutation that either protects it from the action of the drug or neutralizes it ${ }^{7}$. Globally there has been a surge of resistant pathogens including methicillin resistant staphylococcus aureus, penicillin resistant streptococcus pneumonia and vancomycin resistant enterococci. Any micro-organism that survives an antibiotic treatment can multiply and pass on its resistant properties. A factor responsible for this unfortunate scenario includes;

- Over-prescribing and under prescribing antibiotics by the caring physician.

- Exaggeration of symptoms by patients to get a prescription of antibiotics not actually needed $^{5}$

- Reluctance on part of the patients to follow complete course of antibiotics as they feel better $^{6}$

- wrong choice of antibiotics

- Using antibiotics for conditions where they are not indicated such as viral infections (common cold and flu like symptoms)

In the United States, according to a 2013 report by the Centers for Disease Control and Prevention, at least 2 million people annually "acquire serious infections with bacteria that are resistant to one or more of the antibiotics designed to treat those infections." It is estimated that at least 23,000 people die annually from antibiotic-resistant infections. Comparative morbidity and mortality is much higher in under developed and developing nations of the world. The spectrum of resistance differs across developed and developing countries specific to their own circumstances ${ }^{4}$. Pneumococcal resistance towards penicillin and macrolides are the highest in Latin American countries, as is resistance of Salmonella and Escherichia coli towards ampicillin and sulfamethoxazole-trimethoprim ${ }^{2}$

In Pakistan as in many developing countries especially African, Asian and Latin American, antibacterial drugs are grossly misused for reasons cited below;

- There seems to be a culture of self diagnosis and treatment based on previous experiences

- Doctors are keen on emperical use of antibiotics without bothering to determine actual sensitivity of the organisms involved 
- Private practitioner charging handsome fees feel somewhat obliged to prescribe high end antibiotics for patient satisfaction.

- Trend to use antibiotics left over from previous prescriptions in favor of the individual or other house hold members.

- Desiring a quick relief from symptoms without waiting for final diagnosis based on appropriate investigations such as cultures.

- There is a basic lack of awareness ${ }^{8}$ about the ill effects of antibiotic misuse amongst the public and a growing trend of injudicious antibiotic prescription by health professional, lay person and unskilled practitioners.

The increasing number of drug-resistant infections results in:

- crippling illness or disability

- Increased mortality from previously treatable infections

- Prolonged recovery

- Repeated hospitalization with increased length of stay

- More medical consultations

- Less effective and more-invasive treatments

- Higher cost of treatment

A number of important bodies such as the World Health Organization and the British House of Lords have identified the reasons for the emergence of resistance to antimicrobial agents and the preventive measures which need to be urgently implemented to curb the spread of resistant organisms. ${ }^{3}$ It's about time that we in Pakistan should start thinking in terms of Antibiotic stewardship - i.e. appropriate use of Antibiotics. It involves a multifaceted approach to preventing emergence of antimicrobial resistance by selecting an appropriate drug and optimizing its dose and duration to cure an infection while minimizing toxicity ${ }^{9}$. This will help ensure maintaining efficacy and life span of antibiotics. Available data suggest that good antibiotic stewardship reduces rates of Clostridium difficile-associated diarrhea, resistant gram-negative bacilli, and vancomycin-resistant enterococci.

For an Antibiotic Stewardship Program (ASP) to be effective, it should focus on improving quality of care, reducing drug resistance, and ensuring cost effectiveness. For implementation of such a program it is important to understand and allay the concerns of general population and highlight the benefits of the proposed changes. ${ }^{10}$ There is also a need for creating public awareness and imposing diagnostic and therapeutic guidelines at the level of hospitals and other health institutions on lines set out below;

- Doctor prescription should be mandatory for dispensing antibiotics.

- Dosage regimens should be strictly adhered to for the duration prescribed.

- Do away with left over antibiotics

- Discourage the practice of self diagnosis and treatment

- Disregard any previous prescriptions and do not take antibiotics prescribed for any other person

- The age old concept of 'prevention is better than cure' should be followed by practicing basic hygiene principles like hand washing, covering food and vegetables and keeping kitchen surface and utensils clean, at all times.

- Some vaccines protect against bacterial infections, such as diphtheria and whooping cough (pertusis). 
To conclude, we are already witnessing a dip in utility of antibiotics in this post antibiotic era and if this abuse is allowed to progress unchecked we may find ourselves at the mercy of dangerous infections and epidemics that we will struggle to control,

\section{REFERENCES}

1. laniro G, Tilg H, Gasbarrini A (2016) Antibiotics as deep modulators of gut microbiota: between good and evil. Gut gutjnl-2016-312297.

2. English BK, Gaur AH (2010) the use and abuse of antibiotics and the development of antibiotic resistance. Adv Exp Med Biol 659: 73-82.

3. Pechere JC (2001) Patients' interviews and misuse of antibiotics. Clin Infect Dis 33 Suppl 3: S170173.

4. Edwards DJ, Richman PB, Bradley K, Eskin B, Mandell M (2002) Parental use and misuse of antibiotics: are there differences in urban vs. suburban settings? Acad Emerg Med 9: 22-26

5. Soyka LF, Robinson DS, Lachant N, Monaco J (1975) the misuse of antibiotics for treatment of upper respiratory tract infections in children. Pediatrics 55: 552-556.

6. Amábile-Cuevas C (2010) Antibiotic resistance in Mexico: a brief overview of the current status and its causes. J Infect Dev Ctries. Mar 29;4(3):126-31.

7. Naveed S, Qamar F, Maqsood A, Ayub A, Kauser H, Malik H, Fatima K and Hameed A. Prevalence and Consequences of Misuse of Antibiotics, Survey Based Study in Karachi Jinnah University for Women, Karachi, Pakistan. J Bioequiv Availab 2015, 7:5

8. Samaranayake LP, Johnson NW (1999) Guidelines for the use of antimicrobial agents to minimise development of resistance. Int Dent J. Aug; 49(4):189-95.

9. Fishman N Antimicrobial stewardship. Am J Med. 2006 Jun; 119(6 Suppl 1):S53-61; discussion S62-70.

10. Goff DA Antimicrobial stewardship: bridging the gap between quality care and cost. Curr Opin Infect Dis. 2011 Feb; 24 Suppl 1:S11-20.

\section{(c) (i) (-)}

LICENSE: JGMDS publishes its articles under a Creative Commons Attribution Non-Commercial Share-Alike license (CC-BY-NC-SA 4.0). COPYRIGHTS: Authors retain the rights without any restrictions to freely download, print, share and disseminate the article for any lawful purpose. It includes scholarly networks such as Research Gate, Google Scholar, LinkedIn, Academia.edu, Twitter, and other academic or professional networking sites. 